Le Lait, 1988, 68 (4), 435-442

\title{
Application de l'immunologie (anticorps monoclonaux) à la caractérisation d'une souche de Penicillium camemberti Thom
}

\author{
Joëlle DUPONT *, L. POLONELLI ** et G. MORACE ** \\ * Laboratoire de Cryptogamie du MNHN de Paris, \\ 12, rue Buffon, 75005 Paris (UA 257 CNRS), France \\ ** Istituto di microbiologia, Facolta di medicina e chirurgia " A Gemelli », \\ Universita Cattolica del Sacro Cuore, Roma, Italy
}

\section{Résumé}

Des anticorps monoclonaux sont utilisés pour caractériser une souche de Penicillium camemberti au sein d'un groupe de 14 souches de la même espèce.

La méthode d'immunoblotting (Western blot analysis) employée permet le repérage de déterminants antigéniques par des anticorps monoclonaux. La spécificité antigénique est un caractère plus stable que la morphologie ou les activités métaboliques, généralement considérées pour la caractérisation de souches.

Mots clés : Penicillium camemberti - Fromagers - Anticorps monoclonaux - Spécificité antigénique.

\section{Summary}

Characterization of a Penicillium camemberti Thom isolate by monoclonal antibodies.

Monoclonal antibodies are used for the differenciation of a strain of Penicillium camemberti against 13 others of the same species.

The method, Western blot analysis, is based on the antigenic specificity ; this character is more stable than morphology or metabolic activities, generally used for strains characterization.

Key words : Penicillium camemberti - Cheese makers - Monoclonal antibodies - Antigenic specificity.

\section{Introduction}

L'objet de cette étude est de caractériser une souche de Penicillium camemberti au sein d'un groupe de 14 souches de la même espèce, régulièrement utilisées en fromagerie. 
La préoccupation ponctuelle de caractérisation de souche n'a aucune ambition systématique ; elle est seulement destinée à trouver un ou des marqueurs stables pour un organisme appelé à être utilisé dans l'industrie. L'analyse comparée de la morphologie des thalles des 14 souches et de leur vitesse de croissance à diverses températures, ne permet pas de les différencier clairement. Ces critères sont généralement étudiés pour identifier une espèce de Penicillium, ils ne conviennent pas pour travailler au niveau de la souche. D'ailleurs la systématique des Penicillium basée sur ces critères est actuellement remise en question et de nouvelles techniques et approches (SAmSON et PITT, 1985) se développent pour préciser les regroupements existants. Parmi ces techniques nous avons retenu pour comparer nos souches, une méthode immunologique exploitant la spécificité antigénique des organismes (PolonelLI, 1985b).

L'immunologie n'est pas un outil nouveau en agroalimentaire (DAcosta, 1984), et l'application des anticorps monoclonaux se répand, notamment pour la détection des mycotoxines.

Nous avons préféré cette approche qui considère des éléments constitutifs des cellules (les exoantigènes étudiés sont des glycoprotéines) aux comparaisons d'activités enzymatiques (LENOIR et al., 1973) ou de production de métabolites secondaires directement dépendantes des conditions de culture. D'autre part la méthode utilisée dans le présent travail (TowBin et al., 1979) a fait ses preuves dans le domaine médical (Polonelli et al., 1986 a et b). Le principe est de détecter des peptides constitutifs des protéines antigéniques par réaction avec des anticorps monoclonaux ; ces peptides, assimilés à des déterminants antigéniques, sont caractérisés par leur poids moléculaire évalué par comparaison avec des protéines de référence. Les anticorps monoclonaux utilisés ont été sélectionnés et produits par l'équipe du Docteur Polonelli. Pour des raisons pratiques dépendant de la difficulté de production nous avons utilisé des anticorps dirigés contre Microsporum canis. Ils sont dits hétérologues puisqu'ils ne proviennent pas du champignon Penicillium camemberti avec lequel nous les confronterons. L'utilisation d'anticorps monoclonaux homologues (provenant de Penicillium camemberti) n'est d'ailleurs pas indispensable pour le type de réponse que nous attendons : en effet il ne s'agit pas de définir un profil antigénique spécifique de Penicillium camemberti mais de caractériser une souche au sein d'un groupe de la même espèce, c'est-à-dire de rechercher des sérotypes différents.

Polonelli et al. (1986 b) puis Morace et al. (1986) ont expérimenté avec succès l'emploi d'anticorps monoclonaux hétérologues pour définir des sérotypes au sein de dermatophytes puis, plus largement au sein d'hyphomycètes apparentés ou non aux dermatophytes. Ils ont ainsi démontré que les réactions croisées qui existent entre les genres Penicillium et Microsporum sont suffisantes pour permettre l'expérimentation.

\section{Matériel et méthodes}

\section{A. Souches}

Les souches étudiées, numérotées de 1 à 14 sont de provenance commerciale à l'exception de l'une d'entre elles (souche 2) qui est un isolement récent et qui est précisément l'objet de la caractérisation spécifique. 
Morphologiquement, elles correspondent toutes à Penicillium camemberti (PITT, 1979).

\section{B. Anticorps}

La production et les caractéristiques des anticorps sont décrites par Polonelli et MORACE (1985a et 1986a). Les auteurs se sont assurés de la monoclonalité des anticorps et ont défini leur appartenance aux Immunoglobulines de classe G. Nous garderons dans cet article, la nomenclature utilisée par POLONELli et MORACE :

Microsporum canis monoclonal antibody UCSC 3

Microsporum canis monoclonal antibody UCSC 5

Microsporum canis monoclonal antibody UCSC 7

Microsporum canis monoclonal antibody UCSC 13

\section{Méthode}

\section{Extraction des antigènes}

Pour chaque souche, trois cultures en tubes sont nécessaires (cultures sur malt-agar âgées de 4 jours). $8 \mathrm{ml}$ d'une solution de merthiolate de sodium 1/5000 (entraînant la mort des cellules) sont introduits dans chaque tube $(3 \times 8 \mathrm{ml})$; les cultures sont alors mises à incuber pendant 24 heures à température ambiante. Les $24 \mathrm{ml}$ (pour chaque souche) sont ensuite collectés, filtrés sur membrane Millipore, répartis en flacons (à raison de $5 \mathrm{ml} /$ flacon) et concentrés par lyophilisation pendant 24 heures. On obtient ainsi un précipité contenant les exoantigènes, de nature glycoprotéique (de poids moléculaire élevé).

2. Identification des exoantigènes selon la méthode de TowBin (1979) : «Western blot analysis ", reprise par Polonelli et al. (1986 a et b).

Les exoantigènes lyophilisés sont solubilisés dans $0,5 \mathrm{ml}$ de tampon « disruptor

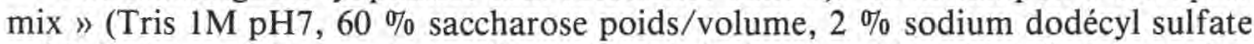
poids/volume, $5 \%$ B mercaptoéthanol poids/volume, $0,02 \%$ bleu de bromophénol en solution saturée). Le mercaptoéthanol présent dans le tampon permet le découpage des protéines en peptides par rupture des ponts disulfurés. Cette étape permet de démasquer des sites inaccessibles du fait de la conformation de la protéine.

Les peptides seront caractérisés par leur poids moléculaire, évalué par référence à des protéines standard de poids moléculaire connu («Pharmacia fine chemicals » Box 175-5-75104 Uppsala 1, Sweden). Les marqueurs sont ajoutés au mélange ; le tout est mis à bouillir pendant 5 minutes.

Les écha itillons sont ensuite déposés sur des gels de polyacrylamide à $10 \%$. L'électroph orèse est réalisée à $20 \mathrm{~mA}$ pendant 18 heures. L'immunoblotting des gels est rualisé selon la méthode de Towbin, modifiée par Polonelli et al. (1986 b). Les peptides sont transférés électriquement sur feuille de nitrocellulose à $60 \mathrm{~V}$ pendant 3 heures (à la moitié du temps, le tampon de transfert est renouvelé). Les feuilles de nitrocellulose sont retirées du système de transfert, séchées à $37^{\circ} \mathrm{C}$ et coupées en bandelettes. Pour chaque souche, nous disposons de plu- 


\section{TABLEAU 1}

Profils antigéniques des souches de Penicillium camemberti obtenus après réaction immunoenzymatique avec les anticorps monoclonaux de Microsporum canis UCSC 3, 5 et 13.

- Souche 2 qui est à caractériser par rapport aux 13 autres souches $\bigcirc$.

Western blot analysis of Penicillium camemberti isolates with Microsporum canis monoclonal antibodies UCSC 3, 5 and 13.

- Isolate 2 which is to characterize against the 13 others $o$.
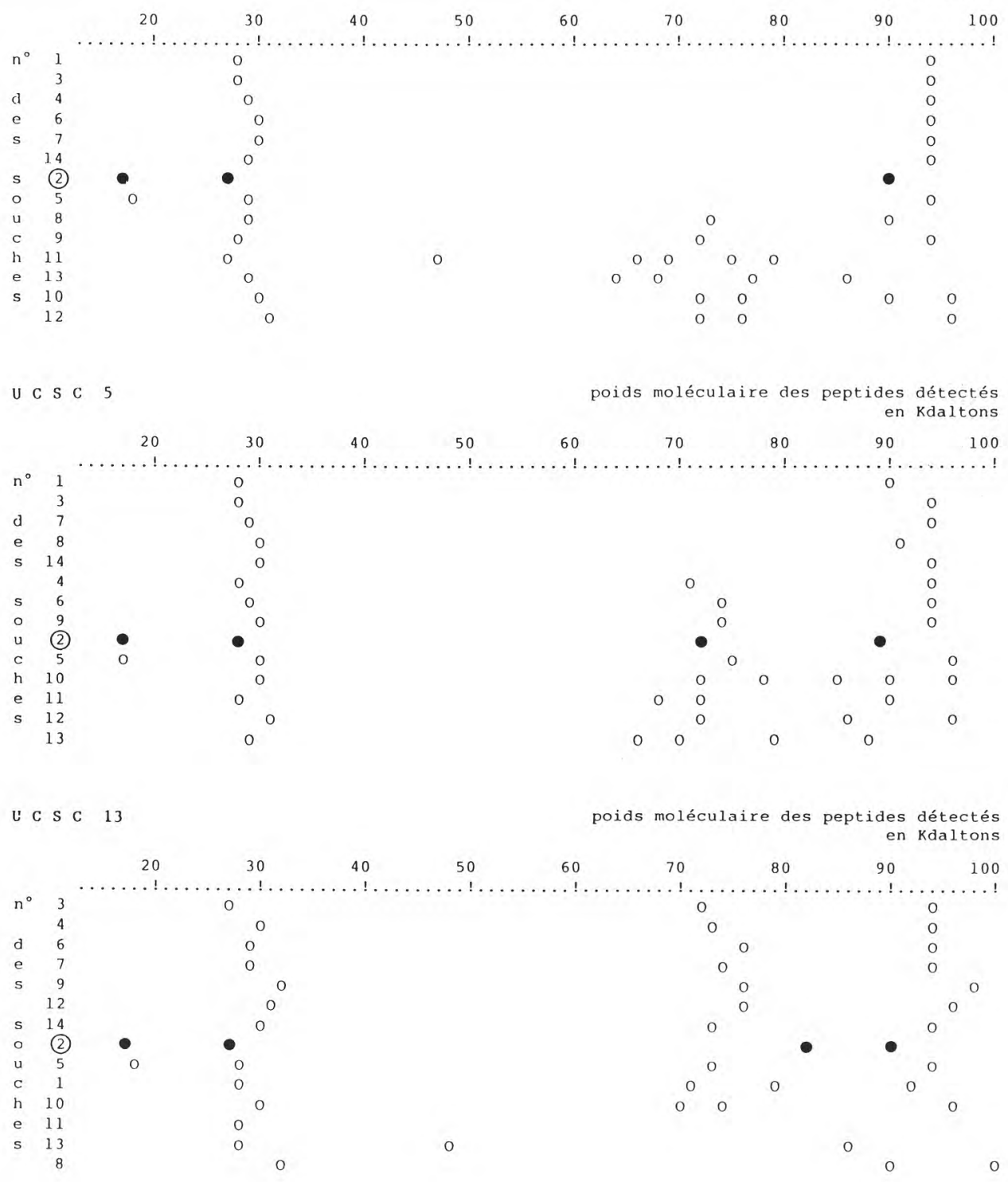
sieurs bandelettes sur lesquelles ont migré de front, les marqueurs protéiques et les peptides antigéniques. Les marqueurs seront révélés séparément au bleu de Coumassie ; leur migration dans le gel permet d'établir une droite étalon des distances de migration en fonction des poids moléculaires (utilisée ultérieurement pour définir le poids moléculaire des peptides).

Les autres bandelettes sont soumises à la réaction immunoenzymatique qui a lieu en deux temps :

- l'anticorps reconnaît les antigènes fixés sur les bandelettes AG-AC1 (AC1 : monoclonal antibody appartenant aux IgG, produit par une souris) ;

- pour identifier l'antigène reconnu par $\mathrm{AC} 1$, on utilise une réaction enzymatique colorée ; une peroxidase est fixée sur le complexe AG-AC1 par l'intermédiaire d'un anticorps AC2 anti AC1 (anti IgG de souris produit par un lapin) : AG-AC1-AC2-peroxidase. Le substrat enzymatique et le colorant sont ajoutés au moment de la révélation.

Pratiquement les bandelettes sont d'abord incubées avec du sérum de cheval à $5 \%$ dans un tampon phosphate (PBS $\mathrm{pH} 7,6$ ) pendant 30 minutes à température ambiante, puis incubées en présence de l'anticorps dilué au $1 / 10$ dans le mélange tamponné de sérum de cheval à $5 \%$ pendant 30 minutes à $37^{\circ} \mathrm{C}$, en agitation. Après rinçage avec du PBS, les bandelettes sont à nouveau mises en contact avec le sérum de cheval à $5 \%$ dans le PBS pH 7,6 pour un second blocage. Enfin elles sont incubées à $37^{\circ} \mathrm{C}$ pendant 30 minutes avec la peroxidase couplée à un sérum de lapin anti IgG de souris, diluée au 1/500 dans le mélange PBS + $5 \%$ sérum de cheval (MILES Y EDTA peroxidase anti- mouse IgG (Rb) CAT 61-204). Après 2 rinçages dans du PBS pendant 30 minutes, les bandelettes sont colorées dans une solution contenant $100 \mathrm{ml}$ d'eau distillée et $1 \mathrm{ml}$ d'une solution à $1 \%$ de 4 chloro l-naphtol et $0,01 \%$ de H202. Des bandes bleues apparaissent : ce sont les peptides que l'on peut assimiler à des déterminants antigéniques, reconnus par l'anticorps monoclonal ; leur poids moléculaire est identifié par référence à la migration des marqueurs protéiques.

\section{Résultats}

La figure 1 montre une photographie d'une bandelette après révélation de la réaction immunoenzymatique. Chaque bande sombre correspond à une réaction de reconnaissance entre un peptide antigénique et l'anticorps monoclonal.

Les peptides reconnus sont répertoriés sur des cartes (tabl. 1 et 2), en fonction de leur poids moléculaire. Ceux de la souche 2 sont représentés par des points noirs - ceux des 13 autres souches sont représentés par des ronds clairs $\bigcirc$.

Deux types de profils apparaissent clairement :

- type A obtenu avec UCSC 3, 5 et 13 (tabl. 1) ;

- type B obtenu avec UCSC 7 (tabl. 2).

Type A :

Peu de déterminants sont reconnus par UCSC 3, 5 et 13 (tabl. 1). Si l'on considère les 3 profils de la souche 2 (repérés par les points obtenus avec les 


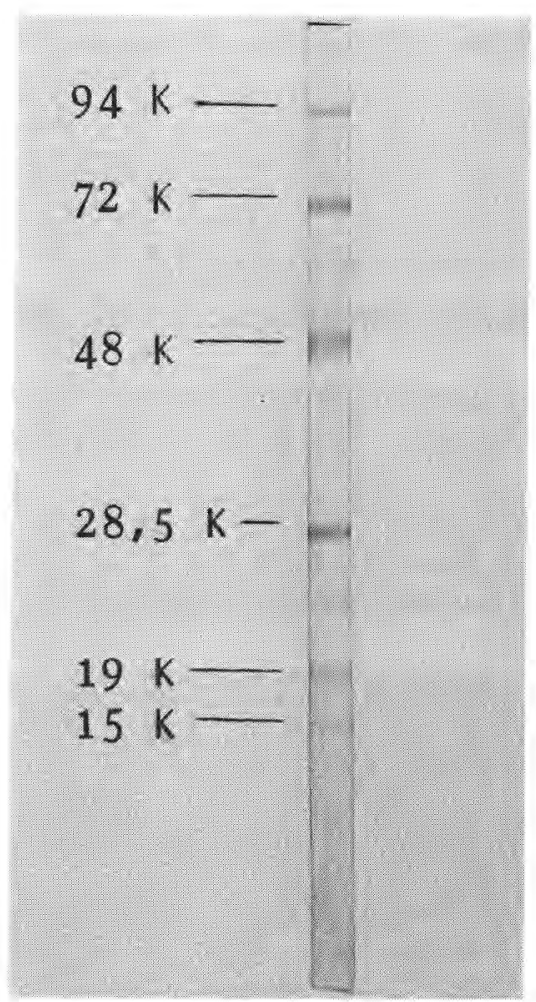

Fig. 1

Exemple d'un immunoblotting des exoantigènes d'une souche de Penicillium camemberti par un anticorps monoclonal.

Western blot analysis of Penicillium camemberti exoantigens by monoclonal antibody.

\section{TABLEAU 2}

Profils antigéniques des souches de Penicillium camemberti obtenus après réaction immunoenzymatique avec l'anticorps monoclonal de Microsporum canis UCSC 7.

Western blot analysis of Penicillium camemberti isolates with Microsporum canis monoclonal antibody UCSC 7.

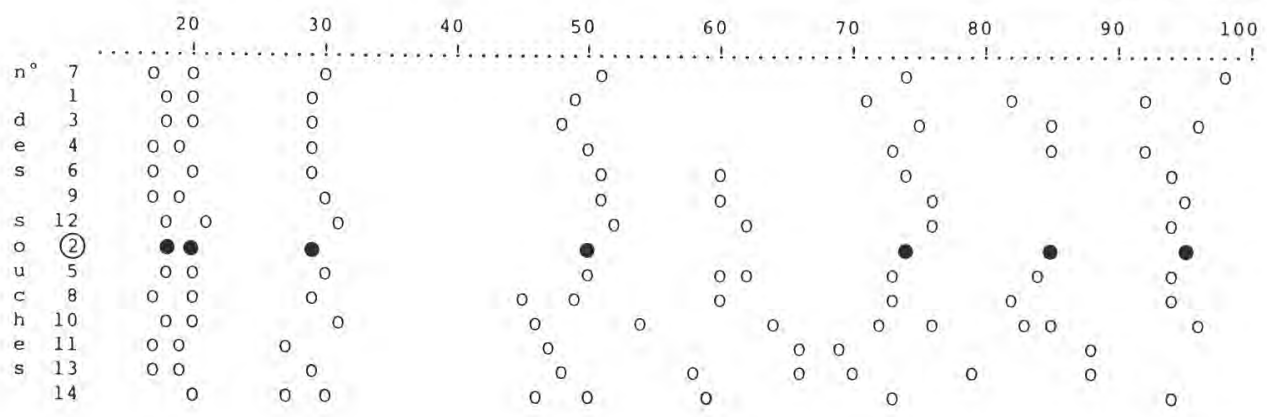


3 UCSC, par rapport aux profils des 13 autres souches (repérés par des points $)$ ), on peut conclure à une grande similitude des souches 2 et 5 , notamment par la présence commune et exclusive du déterminant de $17 \mathrm{Kd}$. La souche 2 ne présente donc pas un profil unique.

Les anticorps monoclonaux UCSC 3,5 et 13 révèlent l'existence de deux sérotypes au sein de nos 14 souches, l'un regroupant les souches 2 et 5 , l'autre regroupant l'ensemble des 12 autres souches.

Type B :

De nombreux déterminants sont révélés après confrontation avec UCSC 7, ce qui permet une comparaison plus fine des souches.

Les déterminants révélés pour la souche 2 sont de $18,20,29,50,74,85$ et 95 $\mathrm{Kd}$; la plupart d'entre eux se retrouvent dans toutes les souches $(18,20,29 \mathrm{Kd})$ ou dans un grand nombre d'entre elles. Dans ce type de profil, c'est plutôt l'absence de déterminants d'environ $60 \mathrm{Kd}$ qui permet d'isoler un groupe de 5 souches : $2,1,3,4$ et 7 .

La confrontation des profils de type A et de type B permet de faire ressortir 2 points :

- la souche 2 , identique à la souche 5 par son profil $\mathrm{A}$, en est dissociée par le profil B ;

- la souche 2 identique aux souches $1,3,4$ et 7 d'après les profils de type $B$, est très différente de ces mêmes souches en regard des profils de type A.

La discrimination de la souche 2 a donc nécessité des confrontations avec plusieurs types d'anticorps monoclonaux.

\section{Conclusion}

Il ressort de la présente étude que la méthode immunologique faisant appel aux anticorps monoclonaux donne des résultats intéressants pour la caractérisation d'une souche donnée dans un ensemble appartenant à la même espèce. Cette possibilité présente un intérêt certain tant au point de vue pratique que juridique avant la diffusion d'une souche dans le domaine industriel.

La rapidité de ce test est un atout considérable.

Du point de vue de l'interprétation des cartes antigéniques, des expériences complémentaires seraient nécessaires pour déterminer si les déterminants antigéniques de poids moléculaires différents sont des modifications ou des états polymérisés d'une seule protéine qui n'auraient pas pu être séparés dans les conditions de dénaturation utilisées dans cette étude. Or notre objectif étant de trouver des différences caractéristiques de la souche 2 par rapport aux 13 autres, nous n'avons pas précisé ni interprété les résultats d'un point de vue immunochimique.

Dans le domaine médical, les anticorps monoclonaux sont déjà employés pour des diagnostics rapides et MORACE et al. (1986) affirme leur intérêt pour des études d'épidémiologie. 
En agroalimentaire, où l'un des soucis primordiaux est de contrôler les matières premières à transformer, notamment en industrie laitière, le développement des techniques immunologiques est tout à fait souhaitable.

Le présent travail est le résultat d'une recherche effectuée à la demande des Fromageries BEL, qui nous ont communiqué les souches.

Reçu le 16 décembre 1986. Accepté pour publication le 20 avril 1988.

\section{Références bibliographiques}

Dacosta Y., 1984. L'immunologie, nouvel outil d'analyse en agroalimentaire. Ed. APRIA. Paris.

Lenoir J., Glenza A., Bergere J.L., Cerf O., Choisy C., Desmazeaud M., Hermier J., 1973. Les facteurs de production du système protéolytique de Penicillium caseicolum. Lait, 53 (525-526), 246-279.

Morace G., Amalfito G., Polonelli L., 1986. Serotyping of fungal isolates by precipitating monoclonal antibodies. Mycopathologia, 94, 53-57.

PITT J., 1979. The genus Penicillium. Academic Press. London.

Polonelli L., Morace G., 1985 a. Serological analysis of dermatophyte isolates with monoclonal antibodies produced against Microsporum canis. J. Clin. Microbiol., 21 (1), 138-139.

Polonelli L., Castagnola M., D’Urso C., Morace G., 1985 b. Serological approaches for identification of Aspergillus and Penicillium species. In : Advances in Penicillium and Aspergillus systematics, ed. SAmSON R.A. and PitT J.I. Plenum Press. New York and London, 267-280.

Polonelli L., Morace G., 1986 a. Specific and common antigenic determinants of Candida albicans isolates detected by monoclonal antibody. J. Clin. Microbiol., 23, 366-368.

Polonelli L., Castagnola M., Morace G., 1986 b. Identification and serotyping of Microsporum canis isolates by monoclonal antibodies. J. Clin. Microbiol., 23, 609-615.

Samson R.A., Pitt J.I., 1985. Advances in Penicillium and Aspergillus systematics. Plenum Press, New York and London.

Towbin H., Staehelin T., Gordon J., 1979. Electrophoretic transfer of proteins from polyacrylamide gels to nitrocellulose sheets : procedure and some applications. Proc. Natl. Acad. Sci. USA, 76 (9), 4350-4354. 